\title{
Journal of Biomaterials
}

\section{Review}

\section{Electrospun Chitosan-based Fibers for Wound Healing Applications}

\author{
Sameer Sapkota, Shih-Feng Chou* \\ Department of Mechanical Engineering, College of Engineering, The University of Texas at Tyler, Tyler, Texas, USA \\ Email address: \\ schou@uttyler.edu (Shih-Feng C.) \\ ${ }^{*}$ Corresponding author
}

\section{To cite this article:}

Sameer Sapkota, Shih-Feng Chou. Electrospun Chitosan-based Fibers for Wound Healing Applications. Journal of Biomaterials. Vol. 4, No. 2, 2020, pp. 51-57. doi: 10.11648/j.jb.20200402.13

Received: November 14, 2020; Accepted: November 25, 2020; Published: December 4, 2020

\begin{abstract}
Chitosan, a natural-occurring biopolymer, is biocompatible to tissues with excellent antibacterial and hemostatic properties, which makes it a great candidate among wound dressing materials. In this paper, electrospun fiber-based wound dressings from blend chitosan and/or polyethylene oxide (PEO) and/or polyvinyl alcohol (PVA) fibers were reviewed. The incorporation of these water-soluble copolymers allows the entanglement of the rigid chitosan molecular chains during electrospinning leading to the production of continuous nonwoven fibers having average diameters ranging from several tenths to hundredths of nanometers. Increasing chitosan composition in the fibers improves the bulk mechanical strength of the fiber mats due to the rigid molecular structure of chitosan. The nano-sized pores within the fiber mats promote permeability of the fiber dressings, which further enhances the exchange of oxygen and nutrients with outside environment. In addition, the porous fiber mat structure facilitates the absorption of wound exudates while reducing the possibility of bacterial infections. Several studies in antibacterial and anti-inflammatory responses of chitosan-based electrospun fibers were discussed in this short review. More importantly, inclusions of small molecule drugs and/or biological agents are possible in chitosan-based electrospun fibers, which provide a multi-purpose treatment capability for wound healing applications.
\end{abstract}

Keywords: Electrospinning, Chitosan, Fibers, Drug Delivery, Wound Healing

\section{Introduction}

Chitosan, a partially $N$-deacetylated derivative of chitin, consists of random mixtures of $\beta$-(1-4)-linked D-glucosamine and $N$-acetyl-D-glucosamine in the polymer backbone. The amount of the D-glucosamine units in chitosan determines the degree of deacetylation (DDA), and DDA has been shown to play an important role on the physicochemical and biological properties of chitosan [1, 2]. Unlike most of other polysaccharides, the amino groups on the D-glucosamine units become protonated making it a cationic polyelectrolyte after dissolving in aqueous acetic acid solution. This unique property allows the molecular chains of chitosan to form electrostatic complexes or multilayered structures with other synthetic polymers having negatively charged functional groups or end caps [3]. In addition, the presence of amine groups on chitosan makes it possible for chemical modifications to include functional groups for biomedical applications [4]. Owning to these special characteristics, various chitosan-based scaffolds have been developed with outstanding mechanical properties, biocompatibility, and biodegradability for tissue engineering applications $[5,6]$.

Among the various tissue engineering scaffolds, chitosan membranes have been developed into dressings for traumatic and/or chronic wounds due to its excellent hemostatic properties, antimicrobial activities, and anti-inflammatory responses [7, 8]. When a wound occurs, platelets become activated and aggregate themselves at the wound site to stop bleeding as the initial response to wound healing, known as the hemostasis stage. At typical wound conditions $(\mathrm{pH}<7)$, chitosan-based membranes are capable of absorbing large amounts of wound exudate and from gelatinous films and/or hydrogels with strong adhesive characteristics to the damaged tissue to stop bleeding [9]. In a clinical study, chitosan gauzes 
were used as a hemostat on 75 patients with traumatic wounds [10]. Results showed that the average wound healing time is comparable to that of commercial dressings, supporting the in vivo histological data on chitosan's ability to induce thrombosis at the tissue surface and promote blood coagulation through platelet activation.

During the hemostasis stage, inflammatory cells, including macrophages and neutrophils, are recruited to the wound site to release a variety of mediators and cytokines that signal the following wound healing events of angiogenesis, thrombosis, fibroblast proliferation, and re-epithelialization. However, unregulated inflammatory responses and/or prolonged bacterial infections may results in the delay of wound healing and the progression of the wounds. It has been shown that chitosan can regulate cellular activity to enable the release pro-inflammatory cytokines and growth factors and to promote the inflammatory microenvironment conducive for healing, such as interleukin 8, prostaglandin E, interleukin $1 \beta$, and others $[9,11]$. In addition to its ability to regulate inflammatory responses, chitosan is also an anti-microbial agent upon gelation after absorbing wound exudates. It is widely accepted that the protonated chitosan molecules attract the negatively charged microbial membrane walls resulting in the membrane wall disassociation followed by the leakage of intracellular electrolytes, which promotes the death of microorganisms [7]. Furthermore, chitosan can increase the paracellular permeability by loosening the cellular tight junctions of the epithelium to enable the delivery of macromolecules (e.g., proteins/peptides and growth factors) to deep tissue for wound healing [12].

The aim of this mini review is to provide the current status on the use of chitosan-based electrospun fibers for antibacterial and anti-inflammation abilities in wound healing. Figure 1 illustrates the scope of this review with the advantages of using chitosan fibers as wound dressings for chronic wounds. In this mini review, compositions of chitosan and the uses of copolymers were discussed with the purpose to provide a short list of possible polymer recipes for electrospinning. In addition, selected works on electrospun chitosan-based fibers were highlighted from the reported data on physico-mechanical properties, antibacterial properties, and anti-inflammation properties of the electrospun chitosan-based fibers. These results suggested that electrospun chitosan-based fibers have the potential to serve as fiber-based dressings to further enable drug delivery and tissue engineering applications. This mini review provides a short but concise discussion on the current status of electrospun chitosan-based fibers for wound healing applications.

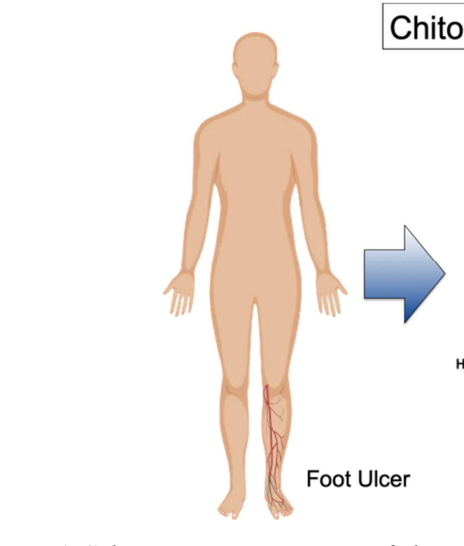

Chitosan Fibers as Topical Dressings in Wound Healing Application
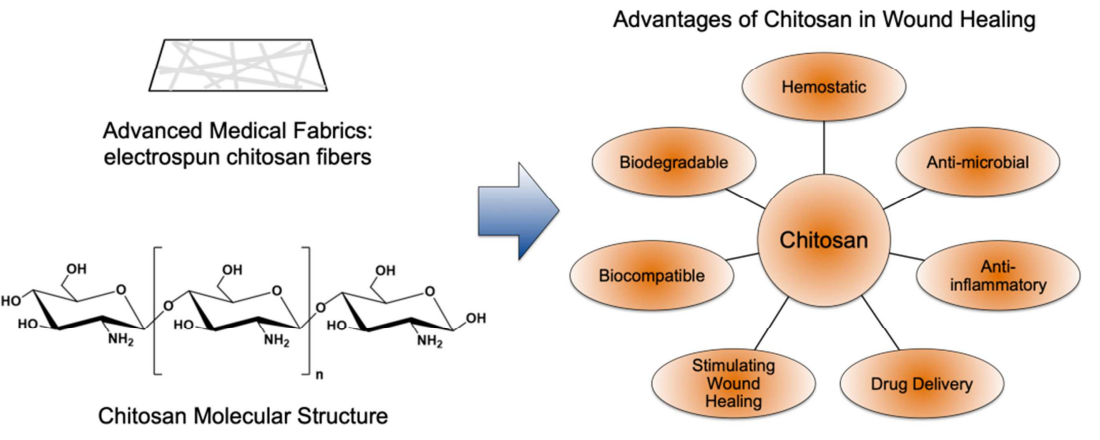

Figure 1. Schematic representation of chitosan-based electrospun fibers in the application of medical fabrics for wound healing applications (e.g., diabetic foot ulcers). The chitosan molecular structure is obtained from [13], an open access article distributed under the CC BY-NC-ND license (http://creativecommons.org/licenses/by-nc-nd/4.0/).

\section{Electrospinning of Chitosan Fibers}

Electrospinning is a straightforward, robust, and versatile method to produce fibers with diameters in the range of a few micrometers to several tenths and/or hundredths of nanometers [14]. The principle of electrospinning, in brief, is to use a strong electric field (e.g., $1 \sim 4 \mathrm{kV} / \mathrm{cm}$ ) to draw solid fibers from a polymer solution. Prior to electrospinning, polymer solutions are prepared with proper solution properties (e.g., viscosity, conductivity, surface tension, and solvent volatility). During electrospinning, the applied electric field needs to overcome surface tension of polymer solution (Taylor cone), where fine strings of fibers are generated and followed by a continuous of "whipping" motion to enable solvent evaporation before landing on the fiber collector. The resulting product is a smooth and dense fiber mat, either in the form of nonwoven or aligned fiber structures with the use of a stationary fiber collector or a rotating drum, respectively. Many have reviewed the effects of electrospinning parameters on the resulting fiber structures with the intent to optimize the electrospinning conditions $[15,16]$.

In order to produce chitosan-based fibrous membranes via electrospinning, it is necessary to blend chitosan with additional polymers. Similar to other naturally occurring polysaccharides (e.g., alginate) [17], the polymer backbones of chitosan consist of rigid blocks of molecular chains in an extended manner due to the repulsive force between ionic groups when dissolved in the aqueous acetic acid solution. This effect results in minimal polymer conformations available, which is required for formation of sufficient chain entanglements to allow continuous fiber formation during electrospinning. Furthermore, formation of strong 
intramolecular hydrogen bonds prevents the free movement of polymeric chain segments that are exposed to the electrical field, which leads to jet break up resulting in electrospraying rather than electrospinning [18]. Both effects make chitosan impossible to electrospin alone, and therefore, chitosan is typically blended with other synthetic polymers to enable chain entanglement and improve chain flexibility to facilitate electrospinning. Currently, published works showed that chitosan could be blended with natural polymers, including gelatin, silk fibroin, and cellulose, as well as synthetic polymers, including polyethylene oxide (PEO), polyvinyl alcohol (PVA), polyethylene terephthalate (PET), and polyesters. Among them, PEO appears to be a good candidate to blend with chitosan for electrospinning, where smooth and uniform fibers can be produced with the ability to modulate drug release rates to deliver biological and/or chemical agents for wound healing.

The incorporation of PEO in chitosan disrupts the self-association of chitosan chains by formation of additional hydrogen bonding between its hydroxyl groups and water molecules. It leads to the reduction in repulsive forces between polycationic groups due to improved chain entanglement, prompting the production of defects free chitosan/PEO nanofiber. Table 1 summarizes electrospinning parameters in studies that use PEO as the copolymer to produce chitosan fibers. According to the study, pure chitosan solution produced spray droplets without forming a fibrous structure due to the high viscosity, whereas the addition of PEO decreased the viscosity of the polymer solution with improvement in producing fibers [19]. Others used $4 \mathrm{wt} \%$ $\mathrm{PEO} /$ chitosan solutions for electrospinning, and the average fiber diameters decreased from several micrometers (PEO/chitosan 100/0) to a few nanometers (PEO/chitosan 60/40) with more beads forming at high chitosan concentration [20]. In another study, PEO/chitosan fibers were prepared from $4-6 \mathrm{wt} \%$ solutions, where ultrafine fibers were produced with a fiber diameter ranged from $80 \mathrm{~nm}$ to $180 \mathrm{~nm}$ [21]. Furthermore, the electrospinnability of PEO/chitosan fibers at various compositions were correlated with the solution properties, including viscosity and $\mathrm{pH}$ level [22]. These fine submicron $\mathrm{PEO} /$ chitosan fibers exhibited high elastic moduli (2.25 MPa) than the cast films (1.19 MPa) [23]. In addition, $\mathrm{PEO} /$ chitosan fibers with a fiber diameter in the range of tenth and hundredth of nanometer favored the antibacterial properties due to high surface to volume ratio [24]. In general, the small fiber diameter, excellent mechanical stiffness, and swelling properties of $\mathrm{PEO} /$ chitosan fibers make them one of the most exciting materials for drug delivery in wound healing.

Table 1. Electrospinning Parameters of various PEO/chitosan Fibers and their Corresponding Fiber Diameters.

\begin{tabular}{|c|c|c|c|c|c|c|c|}
\hline Solvent & $\begin{array}{l}\text { PEO Chitosan } \\
(w t \%)\end{array}$ & PEO/chitosan (v/v) & Voltage $(\mathrm{kV})$ & Distance (cm) & $\begin{array}{l}\text { Flow Rate } \\
(\mathrm{mL} / \mathrm{h})\end{array}$ & $\begin{array}{l}\text { Fiber Diameter } \\
(\mathrm{nm})\end{array}$ & [Ref] \\
\hline $\mathrm{AA} / \mathrm{DMF}^{\mathrm{a}}$ & $\begin{array}{l}3 \% \\
2 \%\end{array}$ & $\begin{array}{l}100 / 0 \\
90 / 10 \\
80 / 20 \\
60 / 40\end{array}$ & $20-25$ & $17-20$ & N/A & $>40$ & [19] \\
\hline $\mathrm{AA}^{\mathrm{b}}$ & $\begin{array}{l}4 \% \\
4 \%\end{array}$ & $\begin{array}{l}100 / 0 \\
90 / 10 \\
80 / 20 \\
60 / 40 \\
40 / 60 \\
20 / 80 \\
0 / 100\end{array}$ & $10-15$ & $10-20$ & 0.5 & $30-200$ & {$[20]$} \\
\hline AA & $\begin{array}{l}1-3 \% \\
1-6 \%\end{array}$ & $\begin{array}{l}1 / 1 \\
2 / 1 \\
5 / 1 \\
1 / 1\end{array}$ & 15 & 20 & 0.1 & $80-180$ & {$[21]$} \\
\hline AA & $\begin{array}{l}\mathrm{N} / \mathrm{A} \\
2 \%\end{array}$ & $\begin{array}{l}1 / 2 \\
1 / 3 \\
1 / 5\end{array}$ & 17 & 10 & 0.1 & N/A & {$[22]$} \\
\hline AA & $\begin{array}{l}2 \% \\
2 \%, 3 \%\end{array}$ & $1 / 1$ & 20 & 10 & 0.3 & $250-500$ & [24] \\
\hline
\end{tabular}

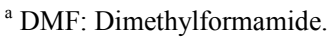

${ }^{\mathrm{b}}$ AA: Acetic acid.

\section{Chitosan Fibers in Wound Healing}

Chitosan, because of its excellent biocompatibility and cationic polyelectrolyte effect at typical wound conditions that provides anti-inflammatory and antimicrobial activities, have a huge potential to be used as fibrous wound dressings. In addition, a case study reported that Celox ${ }^{\mathrm{TM}}$ (chitosan) clotted heparinized blood through the mechanism of direct electrostatic interaction between negatively charged red blood cell membranes and positively charged chitosan [25]. Although chitosan showed great characteristics in wound healing, the use of pristine chitosan fibers in wound dressings is limited due to the poor elastic properties of chitosan. In addition, chitosan is typically dissolved in aqueous acetic acid solutions, whereas it is only partially dissolved in typical wound conditions perhaps due to the solubility limit at the given $\mathrm{pH}$ level. In order to improve the mechanical properties 
and the dissolution behaviors of chitosan-based fibers, it is typically blended with other synthetic polymers into uniformed fibers and/or composites.

Combination of a copolymer for electrospinning of chitosan fibers effectively enables the modulation of fiber physico-mechanical properties, biodegradation, drug release rates, and cellular activities. In a study, chitosan-PEO and fibrinogen fibers were produced simultaneously from two syringe pumps and collected on a rotating mandrel to achieve a composite fiber structure for wound dressing applications [26]. The resulting fiber scaffolds from chitosan-PEO, fibrinogen, and chitosan-PEO/fibrinogen exhibited fiber diameters ranged from $200 \mathrm{~nm}$ to $350 \mathrm{~nm}$, whereas the surface wettability from contact angle studies of the corresponding fibers were $44.2 \pm 5.1^{\circ}, 115.7 \pm 16.2^{\circ}$, and $61.4 \pm 7.6^{\circ}$, respectively. Following the rule of mixture, the average Young's moduli and tensile strength of the chitosan-PEO/fibrinogen fiber scaffolds were $18.84 \pm 5.19$ $\mathrm{MPa}$ and $1.28 \pm 0.38 \mathrm{MPa}$, respectively, as compared to the much higher values of chitosan-PEO fibers and low mechanical properties of fibrinogen fibers. Cellular studies of these fibers loaded with carrier-free recombinant human platelet-derived growth factor-BB (rhPDGF-BB) showed fold differences (approximately 1-fold or less than 1-fold change) in viability of human dermal fibroblasts over 3 days of culture. In particular, cell viabilities were approximately $75 \%$ from fibrinogen fibers as compared to approximately 50\% - 70\% from chitosan-PEO/fibrinogen fibers. In vitro release rate of rhPDGF-BB from chitosan-PEO/fibrinogen fibers at various loading showed sustained release profiles of rhPDGF-BB over 2 days. The study demonstrated the ability to maintain the biological function of rhPDGF-BB throughout the electrospinning process, and introduced the potential use of chitosan-PEO/fibrinogen fiber scaffolds as a viable wound dressing that promote the recruitment of fibroblasts.

Due to the poor electrospinnability of chitosan, blend polymer solutions of chitosan and other copolymers are typically prepared in order to produce uniaxial chitosan fibers. The choice of copolymers plays a major role in determining the fiber properties as a result of the volumetric blend ratios of the polymer, and therefore, may reduce the biocompatibility (toxic) and the stability (biodegradation) of the fibers. To overcome this issue, a hydrophilic derivative of chitosan, carboxymethyl chitosan (CMCS), was used to improve the miscibility with a copolymer (i.e., PEO), a small molecule wound healing drug (i.e., phenytoin sodium), and an antioxidant (i.e., vitamin C) for electrospinning [27]. In vitro release studies from phenytoin sodium loaded and vitamin $\mathrm{C}$ loaded CMCS/PEO fibers (1\% wt/wt $\%$ ) showed $50 \%$ release at approximately $2 \sim 3$ hours followed by $73 \%$ and $68 \%$ release at 48 hours, respectively. In vitro viability assays using mesenchymal stem cells showed that both phenytoin sodium containing and vitamin $\mathrm{C}$ containing $\mathrm{CMCS} / \mathrm{PEO}$ fibers exhibited remarkable cell growth and cell adhesion to the fibrous membranes after 6 days. In vivo rat models on the wound closure rates suggested a $45 \%$ and a $96 \%$ reduction of the wound area for combined phenytoin sodium and vitamin $\mathrm{C}$ loaded CMCS/PEO fibers after 7 and 14 days, respectively. This work suggested the incorporation of a wound-healing drug and an antioxidant on the wound healing ability of electrospun blend modified chitosan/PEO fibers.

To improve the degradability of chitosan fibers, non-biodegradable chitosan fibers were electrospun from blend poly (ethylene terephthalate) (PET) chitosan solutions [28]. The PET/chitosan fibers were crosslinked by glutaraldehyde vapor. Water contact angle studies suggested that all surfaces of various PET/chitosan fibers were hydrophobic with a slight increase of hydrophobicity on those after crosslinking due the diminishing of the free amine groups in chitosan. In vitro viability assays using L929 fibroblasts on the investigation of cytotoxicity compounds released from the fibers showed a rapid cell growth during the first 7 days of culture followed by a stationary phase up to 14 days. Microscopic evaluation on the cell culture showed good cell adhesion at early stage of the culture followed by excellent cell spreading and proliferation with abundant secretion of extracellular matrix (ECM) that partially covered the scaffold surfaces. This work provides information on electrospinning of non-biodegradable (PET/chitosan) fibers that are suitable for the uses of wound dressing membranes to promote cellular activities.

In another study, tetracycline hydrochloride $(\mathrm{TCH})$, an antibiotic used for various infections, was loaded ( $5 \% \mathrm{wt} / \mathrm{wt} \%)$ in electrospun polyvinyl alcohol (PVA)/chitosan fibers for control drug delivery in antibacterial properties [29]. The TCH-loaded PVA/chitosan fibers were crosslinked by glutaraldehyde vapor, where a slight increase in fiber diameter and decrease in surface pores were observed. In vitro $\mathrm{TCH}$ release displayed a burst release behavior with a $75 \%$ cumulative release within 3 hours followed by a plateau of $80 \%$ cumulative release up to 24 hours. The burst release of $\mathrm{TCH}$ was associated with surface accumulation of the drug on the fiber surface due to the low molecular weight of the drug and its affinity to the solvent in electrospinning. The bacterial inhibitory efficacies of TCH-loaded PVA/chitosan fibers $(20$ $\mathrm{mm} \times 10 \mathrm{~mm}$ ) were evaluated by zone of inhibition, where average diameter of the neat inhibition zones were $8.8 \pm 0.4 \mathrm{~mm}$, $15.6 \pm 0.3 \mathrm{~mm}$, and $19.6 \pm 0.2 \mathrm{~mm}$ for E. coli, S. epidermidis, and $S$. aureus. In vitro stretch assays using monolayer of rabbit aortic smooth muscle cells (RMCs) showed no differences between the control groups and the TCH-loaded PVA/chitosan fibers during 24 hours of incubation. Over $90 \%$ of wound closures were found in all groups suggesting the biocompatibility of the TCH-loaded PVA/chitosan fibers without down regulating cell migration ability. Overall, this study suggested a possible strategy in the incorporation of antibacterial drugs in electrospun chitosan fibers as a potential candidate in wound dressing.

Silver (Ag) nanoparticles have shown effectiveness in antibacterial activities, and the delivery of these nanoparticles in solid dosage forms using water-soluble PEO/chitosan fibers further improves the antibacterial ability. Incorporation of the silver nanoparticles in electrospun fibers is typically a two-step process with the use of toxic chemicals as the reduce 
agent and to improve dispersion in polymer solution. Studies demonstrated a one-step process for the production of Ag-PEO/chitosan fibers from UV light irradiation of silver nitrate $\left(\mathrm{AgNO}_{3}\right)$ electrospinning solutions [30]. The $\mathrm{Ag}$ nanoparticles had an averaged size of $3.5 \pm 0.6 \mathrm{~nm}$ analyzed by the transmission electron microscopy that were homogeneously dispersed in PEO/chitosan fibers $(\sim 250 \mathrm{~nm}$ to $350 \mathrm{~nm}$ in diameter) as well as on the fiber surfaces. The average tensile strength of Ag-PEO/chitosan fibers ranged from $5.50 \pm 0.19 \mathrm{MPa}$ to $7.54 \pm 0.74 \mathrm{MPa}$, depending on the $\mathrm{AgNO}_{3}$ concentration in the electrospinning solution. In addition, in vitro release of the $\mathrm{Ag}$ ions showed a fast release rate on the concentration of $\mathrm{Ag}$ ions at $10 \mathrm{~h}$ followed by a slow release that ultimately reached a steady state at $72 \mathrm{~h}$. Antibacterial activities were performed on gram-positive $S$. aureus and gram-negative $E$. coli by zone inhibition methods. Results showed 1.9-fold and 3.4-fold increases of inhibition zones from Ag-PEO/chitosan fibers on $S$. aureus and E. coli as compared to the blank $\mathrm{PEO} /$ chitosan fibers. These fibers were evaluated with cytotoxicity assays using pig iliac endothelial cells, and results suggested over $90 \%$ viability of the cells after $24 \mathrm{~h}$ of culture. This study not only provided a one-step process in the incorporation of Ag nanoparticles in chitosan fibers, but also suggested the wide variety of chemical and biological agents that are compatible with electrospun chitosan fibers.

Several natural ingredients, including olive oil, have been identified as potential wound healing agents prior to the modern medicine. The monounsaturated fatty acids in olive oil have the ability to restore the permeability barrier in skin tissue. In addition, it contains vitamin $\mathrm{E}$ and phenol compounds that are known to for the antioxidant activities. In a study, PEO/chitosan solutions and polycaprolactone (PCL)/olive oil solutions were prepared separately and electrospun together into composite fibers [31]. The composite fibers were further crosslinked by glutaraldehyde vapor, and the $\mathrm{PEO} /$ chitosan and PCL/olive oil composite fibers showed a 0.6 -fold decrease in degree of swelling and 0.3 -fold decrease in weight loss in the release media after $24 \mathrm{~h}$. In vitro release of olive oil from the composite fibers showed a fast release in the first $3 \mathrm{~h}$ followed by a gradual increase toward maximum at $24 \mathrm{~h}$. In vitro cell culture using human dermal fibroblasts showed good cell attachment at early stage of the culture, whereas the nontoxic behavior of the fibers promoted the growth and proliferation of the cells. Antibacterial activity assays using inhibition zones of gram-positive $S$. aureus and gram-negative $E$. coli showed that both pure PEO and pure PCL fibers exhibited no significant antibacterial activities. However, the composite fibers demonstrated significant antibacterial activities against both $S$. aureus and E. coli. In general, this study showed the incorporation of chitosan and natural ingredients in electrospun composite fibers with the ability to down-regulate local bacterial activities that could potentially lead to infection in wound healing.

Table 2. Summary of Drug-eluting Chitosan-based Electrospun Fibers in Wound Healing Applications.

\begin{tabular}{|c|c|c|c|c|}
\hline $\begin{array}{l}\text { Primary } \\
\text { Polymers }\end{array}$ & Additives & Results & Significance & Ref \\
\hline $\mathrm{PEO} /$ Chitosan & $\begin{array}{l}\text { Platelet-derived } \\
\text { growth factor } \\
\text { (PDGF) and } \\
\text { fibrinogen }(\mathrm{Fb})\end{array}$ & $\begin{array}{l}\mathrm{PEO} / \text { chitosan/Fb fibers showed adequate mechanical } \\
\text { properties with acceptable water transfer rates for wound } \\
\text { healing applications. In vitro release of PDGF was effective } \\
\text { at promoting fibroblast migration. }\end{array}$ & $\begin{array}{l}\text { PDGF-loaded } \mathrm{PEO} / \mathrm{chitosan} / \mathrm{Fb} \text { fibers } \\
\text { possessed characteristics that would be } \\
\text { highly beneficial as novel bioactive } \\
\text { dressings for enhancement of wound } \\
\text { healing. }\end{array}$ & [26] \\
\hline PEO/Chitosan & $\begin{array}{l}\text { Phenytoin sodium } \\
\text { (PHT-Na)/Vitamin } \\
\text { C }\end{array}$ & $\begin{array}{l}\text { In vitro drug release rates of the two drugs were similar. Drug } \\
\text { containing PEO/chitosan fibers showed good cell viability. In } \\
\text { vivo animal wound models suggested fibers containing } \\
\text { vitamin } \mathrm{C} \text { and PHT-Na improved wound closure efficiency. }\end{array}$ & $\begin{array}{l}\text { Wound treated with electrospun fibers } \\
\text { containing two drugs regenerated } \\
\text { epidermis layers without necrosis. }\end{array}$ & {$[27]$} \\
\hline Chitosan & $\begin{array}{l}\text { Poly (ethylene } \\
\text { terephthalate) }\end{array}$ & $\begin{array}{l}\text { Improvement on L929 fibroblasts adhesion, spreading, and } \\
\text { superficial proliferation on scaffolds with smaller fiber and } \\
\text { pore diameters. Scaffolds with larger pores enhanced cell } \\
\text { penetration and internal colonization. }\end{array}$ & $\begin{array}{l}\text { Development and optimization of } \\
\text { electrospun scaffolds toward an improved } \\
\text { biological performance. }\end{array}$ & [28] \\
\hline PVA/Chitosan & $\begin{array}{l}\text { Tetracycline } \\
\text { hydrochloride } \\
\text { (TCH) }\end{array}$ & $\begin{array}{l}\text { In vitro TCH release showed a burst delivery }(<2 \mathrm{hrs}) \\
\text { providing an effective antibacterial activity. MTT assays } \\
\text { indicated good cytocompatibility with rabbit aortic smooth } \\
\text { muscle cells. }\end{array}$ & $\begin{array}{l}\text { Incorporation and release of antibiotic } \\
\mathrm{TCH} \text { for wound dressing applications. }\end{array}$ & [29] \\
\hline $\mathrm{PEO} /$ Chitosan & Silver Nitrate & $\begin{array}{l}\text { Ag-loaded PEO/chitosan fibers showed good tensile strength } \\
\text { while exhibiting excellent antibacterial activity and } \\
\text { cytocompatibility. In vitro release showed a sustained release } \\
\text { of silver nanoparticles from fibers ( }>72 \mathrm{hrs} \text { ). }\end{array}$ & $\begin{array}{l}\text { Ag-loaded PEO/chitosan nanofibers } \\
\text { exhibited good potentials in wound } \\
\text { dressings. }\end{array}$ & [30] \\
\hline PEO/Chitosan & PCL/Olive Oil & $\begin{array}{l}\text { In vitro release of olive oil from PEO/CS/PCL fibers showed } \\
\text { a burst release rate }(<3 \mathrm{hrs}) \text {. Fibers were found to have good } \\
\text { attachment, spreading, and proliferation using HDF } \\
\text { fibroblast cells with high antibacterial activities. }\end{array}$ & $\begin{array}{l}\mathrm{PEO} / \text { chitosan/PCL/olive oil composite } \\
\text { fibers had potentials to be ideal wound } \\
\text { dressings. }\end{array}$ & [31] \\
\hline
\end{tabular}

\section{Conclusion}

In general, the excellent antibacterial and hemostatic properties of chitosan make it an ideal candidate for wound dressing materials. The amino groups on the D-glucosamine units can be functionalized to promote drug-polymer and/or polymer-polymer interactions that enable long-term drug 
delivery as well as physicochemical properties modulations of the polymers. Electrospinning of chitosan fibers, however, still remains a technical challenge, where the incorporation of suitable copolymers and the use of non-toxic solvents will play a major role in the development of chitosan-based drug-eluting fibers for wound healing.

\section{Future Directions}

The future directions of electrospun chitosan-based fibers in drug-delivery and wound healing applications include the following potential topics. To promote sustained drug-delivery from chitosan-based fibers, strategies in coupling of wound healing drug molecules with the protonated amino groups on the D-glucosamine units of chitosan may be able to provide a multi-day treatment capacity through drug-polymer interactions. In addition, chitosan may be electrospun with water insoluble polymers, such as polyesters, to provide a more hydrophobic surface to reduce the diffusion and dissolution rate of the drugs from polymer fibers. Furthermore, the production of coaxial fibers with chitosan in the core and water insoluble polymer at the shell is another approach toward sustained drug delivery in wound healing. Finally, chemically modified chitosan has been explored, and chitosan modifications toward better electrospinning ability and/or improved antibacterial and hemostatic properties will be able to advance the field in electrospun chitosan-based fibers for long-term effectiveness in wound healing.

\section{Author Contributions}

All authors contributed equally on the manuscript.

\section{Conflict of Interest}

The authors declare no conflict of interest regarding the publication of this paper.

\section{Acknowledgements}

This work was supported by a grant from the American Heart Association (18AIREA33960372) awarded to S. F. C. Although the funder has sponsored this project, it neither endorses nor rejects the findings of this manuscript.

\section{References}

[1] C.-C. Sun, S.-F. Chou, J.-Y. Lai, C.-H. Cho, C.-H. Lee, Dependence of corneal keratocyte adhesion, spreading, and integrin $\beta 1$ expression on deacetylated chitosan coating, Materials Science and Engineering: C. 63 (2016) 222-230. doi: 10.1016/j.msec.2016.02.069.

[2] S.-F. Chou, J.-Y. Lai, C.-H. Cho, C.-H. Lee, Relationships between surface roughness/stiffness of chitosan coatings and fabrication of corneal keratocyte spheroids: Effect of degree of deacetylation, Colloids and Surfaces B: Biointerfaces. 142 (2016) 105-113. doi: 10.1016/j.colsurfb.2016.02.051.
[3] J. Venkatesan, S.-K. Kim, Chitosan composites for bone tissue engineering-An overview, Marine Drugs. 8 (2010) 22522266. doi: $10.3390 / \mathrm{md} 8082252$.

[4] E. V. R. Campos, J. L. Oliveira, L. F. Fraceto, Poly (ethylene glycol) and cyclodextrin-grafted chitosan: From methodologies to preparation and potential biotechnological applications, Frontiers in Chemistry. 5 (2017) 93. doi: 10.3389/fchem.2017.00093.

[5] S. V. Madihally, H. W. T. Matthew, Porous chitosan scaffolds for tissue engineering, Biomaterials. 20 (1999) 1133-1142. doi: 10.1016/S0142-9612(99)00011-3.

[6] S. Ahmed, Annu, A. Ali, J. Sheikh, A review on chitosan centred scaffolds and their applications in tissue engineering, International Journal of Biological Macromolecules. 116 (2018) 849-862. doi: 10.1016/j.ijbiomac.2018.04.176.

[7] T. Dai, M. Tanaka, Y.-Y. Huang, M. R. Hamblin, Chitosan preparations for wounds and burns: antimicrobial and wound-healing effects, Expert Review of Anti-Infective Therapy. 9 (2011) 857-879. doi: 10.1586/eri.11.59.

[8] H. Liu, C. Wang, C. Li, Y. Qin, Z. Wang, F. Yang, et al., A functional chitosan-based hydrogel as a wound dressing and drug delivery system in the treatment of wound healing, RSC Advances. 8 (2018) 7533-7549. doi: 10.1039/C7RA13510F.

[9] R. Jayakumar, M. Prabaharan, P. T. Sudheesh Kumar, S. V. Nair, H. Tamura, Biomaterials based on chitin and chitosan in wound dressing applications, Biotechnology Advances. 29 (2011) 322-337. doi: 10.1016/j.biotechadv.2011.01.005.

[10] R. A. A. Muzzarelli, P. Morganti, G. Morganti, P. Palombo, M. Palombo, G. Biagini, et al., Chitin nanofibrils/chitosan glycolate composites as wound medicaments, Carbohydrate Polymers. $\quad 70 \quad$ (2007) 274-284. doi: 10.1016/j.carbpol.2007.04.008.

[11] D. Fong, C. D. Hoemann, Chitosan immunomodulatory properties: perspectives on the impact of structural properties and dosage, Future Science OA. 4 (2018) FSO225. doi: 10.4155/fsoa-2017-0064.

[12] M. Amidi, E. Mastrobattista, W. Jiskoot, W. E. Hennink, Chitosan-based delivery systems for protein therapeutics and antigens, Advanced Drug Delivery Reviews. 62 (2010) 59-82. doi: 10.1016/j.addr.2009.11.009.

[13] F. Sami El-banna, M.E. Mahfouz, S. Leporatti, M. El-Kemary, N. A. N. Hanafy, Chitosan as a natural copolymer with unique properties for the development of hydrogels, Applied Sciences. 9 (2019) 2193. doi: 10.3390/app9112193.

[14] S.-F. Chou, D. Carson, K. A. Woodrow, Current strategies for sustaining drug release from electrospun nanofibers, Journal of Controlled Release. 220 (2015) 584-591. doi: 10.1016/j.jconrel.2015.09.008.

[15] V. Beachley, X. Wen, Effect of electrospinning parameters on the nanofiber diameter and length, Materials Science and Engineering: C. 29 (2009) 663-668. doi: 10.1016/j.msec.2008.10.037.

[16] A. Haider, S. Haider, I.-K. Kang, A comprehensive review summarizing the effect of electrospinning parameters and potential applications of nanofibers in biomedical and biotechnology, Arabian Journal of Chemistry. 11 (2018) 11651188. doi: 10.1016/j.arabjc.2015.11.015. 
[17] H. Nie, A. He, J. Zheng, S. Xu, J. Li, C. C. Han, Effects of chain conformation and entanglement on the electrospinning of pure alginate, Biomacromolecules. 9 (2008) 1362-1365. doi: 10.1021/bm701349j.

[18] M. Barchuk, P. Čapková, Z. Kolská, J. Matoušek, D. Poustka, L Šplíchalová, et al., Structure and surface properties of chitosan/PEO/gelatin nanofibrous membrane, Journal of Polymer Research. 23 (2016) 20. doi: 10.1007/s10965-015-0906-0.

[19] N. Bhattarai, D. Edmondson, O. Veiseh, F. A. Matsen, M. Zhang, Electrospun chitosan-based nanofibers and their cellular compatibility, Biomaterials. 26 (2005) 6176-6184. doi: 10.1016/j.biomaterials.2005.03.027.

[20] C.-W. Lou, J.-H. Lin, K.-C. Yen, C.-T. Lu, C.-Y. Lee, Preparation of polyethylene oxide/chitosan fiber membranes by electrospinning and the evaluation of biocompatibility, Textile Research Journal. 78 (2008) 254-257. doi: 10.1177/0040517507089752.

[21] B. Duan, C. Dong, X. Yuan, K. Yao, Electrospinning of chitosan solutions in acetic acid with poly (ethylene oxide), Journal of Biomaterials Science, Polymer Edition. 15 (2004) 797-811. doi: 10.1163/156856204774196171.

[22] J. Vondran, M. Rodriguez, C. Schauer, W. Sun, Preparation of electrospun chitosan-PEO fibers, in: Proceedings of the IEEE 32nd Annual Northeast Bioengineering Conference, IEEE, Easton, PA, USA, 2006: pp. 87-88. doi: 10.1109/NEBC.2006.1629765.

[23] A. Subramanian, D. Vu, G. F. Larsen, H.-Y. Lin, Preparation and evaluation of the electrospun chitosan/PEO fibers for potential applications in cartilage tissue engineering, Journal of Biomaterials Science, Polymer Edition. 16 (2005) 861-873. doi: $10.1163 / 1568562054255682$.

[24] S. G. Kuntzler, J. A. V. Costa, M. G. de Morais, Development of electrospun nanofibers containing chitosan/PEO blend and phenolic compounds with antibacterial activity, International Journal of Biological Macromolecules. 117 (2018) 800-806. doi: 10.1016/j.ijbiomac.2018.05.224.

[25] R. W. J. Millner, A. S. Lockhart, H. Bird, C. Alexiou, A new hemostatic agent: Initial life-saving experience with Celox (chitosan) in cardiothoracic surgery, The Annals of Thoracic $\begin{array}{llll}\text { Surgery. } & 87 & (2009) & \text { e13-e14. }\end{array}$ 10.1016/j.athoracsur.2008.09.046.

[26] T. T. Yuan, A. M. DiGeorge Foushee, M. C. Johnson, A. R. Jockheck-Clark, J. M. Stahl, Development of electrospun chitosan-polyethylene oxide/fibrinogen biocomposite for potential wound healing applications, Nanoscale Research Letters. 13 (2018). doi: 10.1186/s11671-018-2491-8.

[27] M. A. Zarandi, P. Zahedi, I. Rezaeian, A. Salehpour, M. Gholami, B. Motealleh, Drug release, cell adhesion and wound healing evaluations of electrospun carboxymethyl chitosan/polyethylene oxide nanofibres containing phenytoin sodium and vitamin C, IET Nanobiotechnology. 9 (2015) 191200. doi: 10.1049/iet-nbt.2014.0030.

[28] B. Veleirinho, F. V. Berti, P. F. Dias, M. Maraschin, R. M. Ribeiro-do-Valle, J. A. Lopes-da-Silva, Manipulation of chemical composition and architecture of non-biodegradable poly (ethylene terephthalate)/chitosan fibrous scaffolds and their effects on L929 cell behavior, Materials Science and Engineering: $\quad$ C. 33 (2013) 37-46. doi: 10.1016/j.msec.2012.07.047.

[29] A. C. Alavarse, F. W. de Oliveira Silva, J. T. Colque, V. M. da Silva, T. Prieto, E. C. Venancio, et al., Tetracycline hydrochloride-loaded electrospun nanofibers mats based on PVA and chitosan for wound dressing, Materials Science and Engineering: C. 77 (2017) 271-281. doi: 10.1016/j.msec.2017.03.199.

[30] X. Wang, F. Cheng, J. Gao, L. Wang, Antibacterial wound dressing from chitosan/polyethylene oxide nanofibers mats embedded with silver nanoparticles, Journal of Biomaterials Applications. 29 (2015) 1086-1095. doi: $10.1177 / 0885328214554665$.

[31] A. Zarghami, M. Irani, A. Mostafazadeh, M. Golpour, A. Heidarinasab, I. Haririan, Fabrication of $\mathrm{PEO} /$ chitosan/PCL/olive oil nanofibrous scaffolds for wound dressing applications, Fibers and Polymers. 16 (2015) 12011212. doi: $10.1007 / \mathrm{s} 12221-015-1201-8$. 